\title{
BEHAVIOUR OF POINT MASSES AGAINST THE BACKGROUND RADIATION WITH DISSIPATION IN AN EXPANDING UNIVERSE
}

\author{
Toktasyn S. Kozhanov \\ Astrophysical Institute, Kazakh Academy of Sciences \\ 480068 Alma-Ata 68 USSR
}

One of the most important cosmological problems is the question of the properties of systems of particles in a regular field of homogeneous and isotropically expanding two-component Metagalactic substratum [1-7]. Within the framework of the Newtonian cosmology, we consider the behaviour of the ensemble of point masses embedded in a homogeneous expanding medium having two different equations of the state. Dissipation phenomena caused by the chaotic motion of the background matter are taken into account.

Let us study the evolution of the perturbations of the particle density against the background of a homogeneously and isotropically expanding universe with zero pressure, supposing that gravitational influence of the radiation is not essential. The equation of the perturbed motion of particles in the presence of a braking force is of the form

$$
\ddot{r}=-\frac{4}{3} \pi G \rho\left(1+3 \frac{\delta r}{r}\right) r-K \rho V=-\frac{4}{3} \pi G \rho\left(1+3 \frac{\delta r}{r}\right) r-K \rho V,
$$

where $K=\operatorname{const}(K>0)$ is a coefficient of proportionality, $\mathrm{G}$ is the gravitational constant, and $\mathrm{V}$ is the peculiar velocity of particles.

We introduce the following change of variable:

$$
r=R(t)[1-x(t)]
$$

where $x<<1$ characterizes the perturbation of the density and $\mathrm{R}$ is the expansion scale of the universe satisfying Friedman's equation for the matter with zero pressure (Einstein-de Sitter model [6]), e.g. $\rho_{B}=3(R / R)^{2} / 8 \pi G, R / R=2 / 3 t$. Then equation (1) in a linear approximation acquires the form

$$
\dot{x}+(2 \dot{R} / R+K \rho) \dot{x}=4 \pi G \rho x .
$$

The general solution (when $\rho=\rho_{B}, \dot{R} / R=2 / 3 t$ ) is

$$
X(t)=A t^{2 / 3} \exp (K / 6 \pi G t)+B t^{-1} F(1,8 / 3, K / 6 \pi G t)
$$

where $F(a, c, y)$ is a degenerate hypergeometric function [8] and A and B are constants of integration. From (4) it follows that the force of resistance of the expanding matter with zero pressure gives an impulse to the growth of a perturbation density.

Let us take into account the brake action of the matter caused by the radiation in cases when the matter becomes neutral. In models where the radiation with pressure $P=\rho_{r} C^{2} / 3$ prevails, we have $\dot{R} / R=1 / 2 t[6]$, and then the equation (3) acquires the form:

$$
\ddot{x}+\left(\frac{1}{t}+\frac{\bar{\gamma}}{t^{2}}\right) \dot{x}=\frac{3}{8 t^{2}} x \text {. }
$$

The general solution is

$$
x(t)=A_{1} t^{\sqrt{3 / 8}} F_{1}(-\sqrt{3 / 8}, 1-2 \sqrt{3 / 8}, \bar{\gamma} / t)+B_{1} t^{-\sqrt{3 / 8}} F_{2}\left(\sqrt{3 / 8}, 1+2 \sqrt{3 / 8}, \frac{\bar{\gamma}}{t}\right),
$$

where $A_{1}$ and $B_{1}$ are constants of integration and $(\bar{\gamma} / t)<<1$. The change in perturbation density does not radically differ from that in the absence of dissipation forces.

Let us consider the behaviour of a perturbation density of the matter with zero pressure on the strictly homogeneous background radiation with pressure $P=\rho_{r} C^{2} / 3$ with the condition that the gravitational action of baryons in the universe and the gravitational action of the radiation may be equally essential. If the baryons and the radiation do not interact, then the 
equation of perturbed motion of the matter on the nonperturbed expanding background radiation, taking into account the friction force stipulated by the field of radiation, is of the form

$$
\ddot{r}=-\frac{4}{3} \pi G\left[2 \rho_{r}+\rho_{B}\left(1+3 \frac{\delta r}{r}\right)\right] r-p_{r} V,
$$

where $\rho_{r}$ is the density of radiation and $\rho_{B}$ is the density of the matter. If we again use equation (2) to change variables, and if we impose the condition that $R$ satisfies Friedman's equation $R=-(4 / 3) \pi G\left(2 \rho_{r}+\rho_{B}\right) R$, then in a linear approximation, equation (7) becomes

$$
\ddot{x}+\left(2 \dot{R} / R+\gamma p_{r}\right) \dot{x}-4 \pi G \rho_{B} x=0 .
$$

The ratio of the second term to the first $\geqq 30$ in epochs when the redshift $Z \geqq 1000$. This means that in the left side of the equation (8) radiation drag prevails. In this case, taking into account the change of density $\rho_{B}=\left(1 / 6 \pi G t^{2}\right)$ and $\rho_{r}=3 / 32 \pi G t^{2}$ for a flat cosmological model [6], we have from equation ( 8 )

$$
\ddot{x}+\bar{\gamma} / t^{2} \cdot \dot{x}=2 x / 3 t^{2}, \bar{\gamma}=3 \gamma / 32 \pi G .
$$

The general solution of this equation has the form:

$$
x(t)=A_{2} t^{\frac{1}{2}+\sqrt{11 / 12}} F_{1}\left[-\frac{1}{2}-\sqrt{\frac{11}{12}}, 1-2 \sqrt{\frac{11}{12}}, \frac{\bar{\gamma}}{t}\right]+B_{2} t^{\frac{1}{2}-\sqrt{\frac{11}{12}}} F_{2}\left[-\frac{1}{2}-\sqrt{\frac{11}{12}}, 1+2 \sqrt{\frac{11}{12}}, \frac{\bar{\gamma}}{t}\right),
$$

where $A_{2}$ and $B_{2}$ are constants of integration. In this case the density of the matter perturbation is growing faster than the expansion scale of the universe with pressure $P=\rho_{r} C^{2} / 3$.

If the argument of the degenerate hypergeometric function $F(a, c, y)$ tends to large values (which is possible at large $\tau=($ Const $/ \pi G)>0$ and small $t$ ), the amplitude of inhomogeneities grows according to the following laws: in the first case $t^{2 / 3} e^{\tau / t}$, in the second case $t e^{\tau / t}$, and in the third case $t^{2} e^{\tau / t}$.

We conclude that one of the mechanisms promoting the growth of perturbation densities of matter that leads to the strong inhomogeneity of the matter distribution observed at present is the action of the dissipation force (dynamic friction force) arising from the background radiation.

\section{REFERENCES}

1. Lifschitz, E.M. 1946, JETF (Soviet Phys. J.), 16.2, 587.

2. Bonnor, W.B. 1957, Monthly Not. Roy. Ast. Soc., 117.1, 104.

3. Kodama, H. and Sasaki, M. 1984, Progress of Theor. Phys. Supplement. 78, 166.

4. Guyot, M. and Zeldovich, Y.B. 1970, Astron. \& Ap., 9.2, 227.

5. Mazaros, P. 1974, Astron. \& Ap., 37.2, 225.

6. Zeldovich, Y.B. and Novikov, I.D. 1975, Structure and Evolution of Universe, Moscow, "Nauka", 736.

7. Peebles, P.J.E. 1983, The Large-scale Structure of the Universe, Moscow, "Mir", 408.

8. Bbitmen, A. and Ardein, A. 1973, Higher Transcendental Functions, Moscow, "Nauka", 294.

9. Peebles, P.J.E. 1975, Physical Cosmology, Moscow, "Mir", 310. 